\title{
Inclusion of virtual nuclear excitations in the formulation of the $\left(\mathrm{e}, \mathrm{e}^{\prime} \mathrm{N}\right)$ reaction.
}

\author{
G. H. Rawitscher \\ Physics Department, University of Connecticut, Storrs, CT 06268 \\ bigskip
}

A wave-function framework for the theory of the (e,e'N) reaction is presented in order to justify the use of coupled channel equations in the usual Feynman matrix element. The overall wave function containing the electron and nucleon coordinates is expanded in a basis set of eigenstates of the nuclear Hamiltonian, which contain both bound states as well as continuum states.. The latter have an ingoing nucleon with a variable momentum $Q$ incident on the daughter nucleus as a target, with as many outgoing channels as desirable. The Dirac Eqs. for the electron part of the wave function acquire inhomogeneous terms, and require the use of distorted electron Green's functions for their solutions. The condition that the asymptotic wave function contain only the appropriate momentum $Q_{k}$ for the outgoing nucleon, which corresponds to the electron momentum $k$ through energy conservation, is achieved through the use of the steepest descent saddle point method, commonly used in three-body calculations. 


\section{INTRODUCTION.}

The commonly accepted starting point for writing down the (e,e'N) matrix element is a Feynman diagram. The Feynman diagram consists of two vertices: one in which a nucleon is detached from the target nucleus, and the other which represents the electron-nucleon interaction, which scatters the nucleon into the appropriate final state. In 1970 Gross and Lipperheide [四] wrote down such a matrix element, and Boffi and collaborators [2] based their work [3] on this approach. Walecka and collaborators [4], as well as Donnelly and co-workers [5] also start from this type of approach. Additional refinements were gradually introduced. The optical model, initially used to describe the nucleon-nucleus distortion [2], was later refined by using the Random Phase Approximation for the nuclear excitations [6], electron spin variables were introduced [5], the relativistic nucleon-nucleus interaction was considered [7], and two-step processes (or coupled channel effects) were included [8], [9]. Many good reviews of the (e,e’N) formalism exist, the latest one being due to Kelly [10].

The objective of the present paper is to formulate the (e,e' $\mathrm{N})$ reaction in terms of a wave function expansion, rather than Feynman-diagram matrix elements This treatment permits one a) to understand the assumptions which underlie the coupled-channel treatment of the hadron-nucleus channels, which are commonly inserted into the Feynman matrix elements, b) to include the effect of the (e,e' N) channels into the treatment of dispersion corrections to electron-nucleus scattering, c) to provide a foundation for the treatment of the (e,e' $2 \mathrm{~N})$ processes.

The wave function method described here is similar in nature to the expansions employed in three-body reaction theory [11], [12], [13]. In particular, the saddle-point method is used to obtain the asymptotic limit of the wave function for the two particles in the continuum (the electron and the emitted nucleon), thus making contact with the matrix element written

conventionally for the Feynman diagram mentioned above. However, the present formulation 
avoids the Faddeev treatment by neglecting the electro-magnetic interaction between the two particles in the continuum, since it is weak. In the extension to the (e,e' $2 \mathrm{~N})$ case, the interaction between the two nucleons in the continuum being strong, has to be included. This can be achieved by means by a method similar to the continuum discretized coupled channel (CDCC) treatment [14], [15] of the breakup in deuteron-nucleus scattering, still without using the Faddeev approach. However, due to the complexity of the description of the two-nucleon correlations, both in the initial and final states, the extension to the (e,e' $2 \mathrm{~N})$ case is left to a future paper.

Our treatment is as follows. The overall wave function, which contains both the coordinates of the electron and the nucleons, is expanded on a basis set of nuclear states which contain as much of the correlations as needed. In the continuum, these nuclear states contain both ingoing and outgoing waves, even though the physical boundary conditions require that only outgoing nucleon waves exist asymptotically in the overall wave-function. This requirement is satisfied, as is explained below. The coefficients of the expansion of the overall wave function into this basis set are functions of the electron coordinates, which in turn obey inhomogeneous Dirac equations. The latter are solved by employing relativistic distorted Green's functions, as is described in Appendix A. This discussion is presented in Section 2 by means of a simple, one-nucleon example. The generalization to the manynucleon case is done in section 3, and the coupled channel formulation is presented in section 4. Complications due to the non-orthogonality between the bound states and one-nucleonin-the-continuum-states do not arise at this stage because in the original expansion both the bound and the continuum nuclear states are eigenfunctions of the same hamiltonian. This argument is not invalidated by the subsequent replacement of many of the channels by effective complex interactions, such as by T-matrices or optical potentials. The nonorthogonality between the bound and the continuum optical model wave functions is thus an artifact of the optical model approximation, but does not invalidate the overall theory of the $\left(\mathrm{e}, \mathrm{e}^{\prime} \mathrm{N}\right)$ reaction.

The treatment presented here is schematic. The electron-nucleon interaction contains 
many intricate questions which have been addresses before [0] but which are left out here. Also the details of the relativistic Dirac description of the electron, some of which are included in Appendix A, are left out in the text of this paper. Spin and orbital angular momentum information is also left out. In a subsequent paper by J. Kelly on the use of coupled equations in (e,e' p) reactions, some of the details are included explicitly. The questions of principle addressed in this paper are: i) what set of basis states are suitable in the description of the wave functions involved, ii) how can one be sure that in the final state only the outgoing parts of these wave functions survive, and iii) what are the corresponding coefficients (the T-matrix elements) in the asymptotic waves. The realization that only outgoing waves survive for both particles is achieved naturally through the use of the steepest descent method in the evaluation of a saddle point in the integration space. Such approaches are common in the three-body literature, [13, [14 but the extension to the coupled channel case requires additional thought, which is provided here.

\section{THE BASIC IDEAS.}

The basic ideas used in the present approach are illustrated in this section by means of a simplified example. In this model the "daughter" nucleus is described by an inert core, and the target nucleus consists of one valence nucleon bound to this core. The interaction between the electron with the core, and the nucleon with the core, is represented by two mean field potentials $V_{E}\left(r_{e}\right)$ and $V_{N}\left(r_{n}\right)$, respectively. The interaction between the electron and the hadron is denoted by $v_{e n}^{(E)}$. It is commonly expressed in the form $A_{\mu} j_{\mu}$, where $j_{\mu}$ is the electron current and $A_{\mu}$ is the vector potential produced by the nuclear current. The two mean field potentials are real.

The overall wave function is first expanded in a set of partial waves

$$
\Psi\left(\vec{r}_{e}, \vec{r}_{n}\right)=\frac{1}{r_{e} r_{n}} \sum_{J, M_{J}} \psi_{J, M_{J}}\left(r_{e}, r_{n)} \mathcal{Y}_{J, M_{J}}\left(\hat{r}_{e}, \hat{r}_{n}\right)\right.
$$

and each radial wave is expanded into a complete set of nuclear basis states $\phi$ 


$$
\psi\left(r_{e}, r_{n)}=\sum_{i=1}^{B} \chi_{i}\left(r_{e}\right) \phi_{i}\left(r_{n}\right)+\int_{0}^{\infty} \chi_{k_{Q}}\left(r_{e}\right) \phi_{Q}^{(-)}\left(r_{n}\right) d Q\right.
$$

In the above equation spin and angular momentum numbers $J, M_{J}$ should have been included, but, they already are left out.

The discrete functions $\phi_{i}\left(r_{n}\right)$ are radial eigenstates with negative energies of the nucleon Hamiltonian

$$
\left[-\frac{\hbar^{2}}{2 m} \frac{d^{2}}{d r^{2}}+V_{N}(r)\right] \phi_{i}(r)=\epsilon_{i}^{N} \phi_{i}(r), i=1,2, \ldots B
$$

and the positive energy eigenstates form a continuum

$$
\left[-\frac{\hbar^{2}}{2 m} \frac{d^{2}}{d r^{2}}+V_{N}(r)\right] \phi_{Q}^{(-)}(r)=\frac{\hbar^{2} Q^{2}}{2 m} \phi_{Q}^{(-)}(r) \quad 0 \leq Q<\infty .
$$

The functions $\phi_{Q}^{(-)}(r)$ have ingoing as well as outgoing waves. Asymptotically they are given by

$$
\phi_{Q}^{(-)}(r) \approx e^{-i \eta} \sin (Q r+\eta)=\frac{1}{2 i}\left(H^{(+)}(Q r)-H^{(-)}(Q r) e^{-2 i \eta}\right)
$$

The $H^{( \pm)}$'s are related to the outgoing and ingoing Hankel functions according to $H^{(+)}(z)=$ $i z h^{(1)}(z)$, and $H^{(-)}(z)=-i z h^{(2)}(z)$, where the functions $h^{(1,2)}$ are defined in 10.1.1 of [16], and $\eta$ is a nuclear phase shift determined by $V_{N}(r)$. The orthogonality properties of the $\phi$ 's are as follows:

$$
\begin{gathered}
<\phi_{Q}^{(-)} \mid \phi_{Q^{\prime}}^{(-)}>=\int_{0}^{\infty} \phi_{Q}^{(+)}(r) \phi_{Q^{\prime}}^{(-)}(r) d r=\frac{\pi}{2} \delta\left(Q-Q^{\prime}\right) \\
<\phi_{Q}^{(+)} \mid \phi_{Q^{\prime}}^{(-)}>=\int_{0}^{\infty} \phi_{Q}^{(-)}(r) \phi_{Q^{\prime}}^{(-)}(r) d r=e^{-2 i \eta} \frac{\pi}{2} \delta\left(Q-Q^{\prime}\right) \\
<\phi_{Q}^{( \pm)}\left|\phi_{i}>=0 ;<\phi_{i^{\prime}}\right| \phi_{i}>=\delta_{i, i^{\prime}} .
\end{gathered}
$$

In the above,

$$
\phi_{Q}^{(+)}(r)=\left[\phi_{Q}^{(-)}(r)\right]^{*}
$$


The orthogonality between the continuum states and the bound states follows from the fact that the potential $V_{N}$ in Eqs. (2a) and (2b) is the same, hence non-orthogonality is no problem here.

The functions $\chi$, coefficients of the expansion (2.1), are to be determined from their respective Dirac Eqs., as will now be described. The Hamiltonian of the system is

$$
\mathcal{H}=H_{e}+V_{E}+T_{n}+V_{N}+v_{e n}
$$

where $H_{e}$ and $T_{n}$ are the relativistic and non-relativistic kinetic energy operators acting on the electron and nucleon, respectively. The equations for the electron functions $\chi\left(r_{e}\right)$ are obtained by multiplying the radial part of the overall Schrödinger Eq. $(\mathcal{H}-E) \psi\left(r_{e}, r_{n}\right)=0$ on the left with either $<\phi_{i}\left(r_{n}\right) \mid$ or $<\phi_{Q}^{(-)}\left(r_{n}\right) \mid$, making use of Eqs. (2.2) and (2.4), and integrating over $r_{n}$. One obtains

$$
\begin{gathered}
\left(H_{e}+V_{E}-E_{i}^{(e)}\right) \chi_{i}\left(r_{e}\right)=-B_{i}\left(r_{e}\right), \\
\left(H_{e}+V_{E}-E_{k_{Q}}^{(e)}\right) \chi_{k_{Q}}\left(r_{e}\right)=-B_{Q}\left(r_{e}\right),
\end{gathered}
$$

where the inhomogeneous driving terms $B$ are given by

$$
\begin{array}{r}
B_{i}\left(r_{e}\right)=<\phi_{i} \mid v_{e n}^{(E)} \psi\left(r_{e}, r_{n}\right)> \\
B_{Q}\left(r_{e}\right)=<\phi_{Q}^{(-)} \mid v_{e n}^{(E)} \psi\left(r_{e}, r_{n}\right)>.
\end{array}
$$

If one inserts the expansions (2.1) for $\psi\left(r_{e}, r_{n}\right)$ into Eqs. (2.7), then Eqs. (2.6) become integro- differential equations for the functions $\chi_{i}$ and $\chi_{k}$. However, with the exception of the ground state term $\chi_{g}\left(r_{e}\right) \phi_{g}\left(r_{n}\right)$ (here $i=g$ ), which is present in the incident channel, all other $\chi$-terms are of order $v_{e n}$, and hence the inhomogeneous terms $B$ in Eqs. (2.6) are of second order in $v_{e n}$, and they can be neglected as being dispersion correction terms. We follow this procedure here and replace $\psi\left(r_{e}, r_{n}\right)$ by $\psi_{I}$

$$
\psi\left(r_{e}, r_{n}\right) \Rightarrow \psi_{I} \equiv f_{g}^{(+)}\left(r_{e}\right) \phi_{g}\left(r_{n}\right)
$$


in the expressions (2.7) for the $B$ 's. Here $f_{g}^{(+)}$is a spinor which satisfies

$$
\left(H_{e}+V_{E}-E_{g}^{(e)}\right) f_{g}^{(+)}\left(r_{e}\right)=0 .
$$

It has both incident and outgoing waves and describes the scattering of an electron from the core potential,

Regardless wether the replacement (2.8) is made or not, the solutions of Eqs. (2.6) have, by construction, only outgoing waves asymptotically

$$
\begin{gathered}
\chi_{i} \approx T_{i}^{(e)} \exp \left(i \theta_{i}\right) \\
\chi_{k_{Q}} \approx T_{k_{Q}}^{(e)} \exp \left(i \theta_{k_{Q}}\right)
\end{gathered}
$$

where

$$
\begin{gathered}
\theta_{i}=k_{i} r_{e}+\gamma_{i} \ln \left(2 k_{i} r_{e}\right) \\
\theta_{k_{Q}}=k_{Q} r_{e}+\gamma_{k_{Q}} \ln \left(2 k_{Q} r_{e}\right) .
\end{gathered}
$$

In the above expression the $\gamma$ 's are the Sommerfeld parameters, as explained in Appendix A, and the angular momentum phases $l \pi / 2$ have been left out. The factors $T$ in Eqs. (2.10) are spinors which are obtained from the solution of inhomogeneous Dirac equations, as is discussed in Appendix A.

The asymptotic expression for $\psi\left(r_{e}, r_{n}\right)$ is obtained by inserting (2.10) into Eq. (2.1)

$$
\begin{aligned}
\psi\left(r_{e}, r_{n}\right) \approx & \sum_{i=1}^{B} T_{i}^{(e)} \exp \left(\theta_{i}\right) \phi_{i}\left(r_{n}\right) \\
& +\int_{0}^{\infty} T_{k_{Q}}^{(e)} \exp \left(\theta_{k_{Q}}\right) \frac{1}{2 i}\left(H^{(+)}\left(Q r_{n}\right)-H^{(-)}\left(Q r_{n}\right) e^{-2 i \eta}\right) d Q .
\end{aligned}
$$

The electron and nucleon momenta in the expression above are related by energy conservation, and that is what produces the correlation between the two wave functions. Defining the total energy as $E$, the electron energy as $E_{k}^{(e)}=\sqrt{(\hbar c k)^{2}+\left(m_{e} c^{2}\right)^{2}}$, and the kinetic energy of the nucleon as either $\epsilon_{i}^{(N)}$ or $E_{Q}^{(N)}=(\hbar c Q)^{2} / 2 m$, then the conservation of energy equations are 


$$
\begin{gathered}
E_{i}^{(e)}+\epsilon_{i}^{(N)}=\sqrt{\left(\hbar c k_{i}\right)^{2}+\left(m_{e} c^{2}\right)^{2}}+\epsilon_{i}^{(N)}=E \\
E_{k_{Q}}^{(e)}+\epsilon_{Q}^{(N)}=\sqrt{\left(\hbar c k_{Q}\right)^{2}+\left(m_{e} c^{2}\right)^{2}}+(\hbar c Q)^{2} / 2 m=E,
\end{gathered}
$$

from which it follows that $d Q / d k=-(k / Q)\left(m c^{2} / \epsilon_{k}\right)$. Accordingly, the first term $\exp \left(i \theta_{k_{Q}}\right) H^{(+)}$in $(2.12)$, which gives rise to $\exp i\left(r_{e} k+r_{n} Q\right)$, has a stationary phase point at $Q_{0}$, given by

$$
Q_{0} / k_{0}=\left(r_{n} / r_{e}\right)\left(m c^{2} / \epsilon_{k}^{(e)}\right)
$$

and the corresponding integral has a non-vanishing value which can be evaluated by the saddle-point method [17], while $\exp \left(\theta_{k_{Q}}\right) H^{(-)}$has no such point and does not contribute. Using Eqs. (2.13), and neglecting the rest energy of the electron in comparison to the total energy, one obtains

$$
\begin{aligned}
Q_{0} & \simeq\left(r_{n} / r_{e}\right) m c^{2} / \hbar c \\
\hbar c k_{0} & \simeq E-\left(r_{n} / r_{e}\right)^{2} m c^{2} / 2,
\end{aligned}
$$

where $m$ is the nucleon mass. As described in Appendix A, the result is

$$
\begin{gathered}
\int_{0}^{\infty}\left[T_{k_{Q}}^{(e)} \exp \left(\theta_{k_{Q}}\right) \frac{1}{2 i} H^{(+)}\left(Q r_{n}\right) d Q\right. \\
\approx \\
\frac{(i)^{3 / 2}}{2} \frac{r_{n}^{2}}{r_{e}^{5 / 2}} \sqrt{2 \pi m c^{2} /(\hbar c)} T_{k_{0}}^{(e)} \exp \left(\theta_{k_{0}}\right) H^{(+)}\left(Q_{0} r_{n}\right) .
\end{gathered}
$$

The above result shows that asymptotically the wave function contains outgoing waves in both coordinates $r_{e}$ and $r_{n}$ in spite of the fact that the basis set in $r_{n}$ contained ingoing and outgoing waves, and further, that $r_{n}$ and $r_{e}$ are not independent of each other. Rather, the choice of their ratio determines the value of the electron energy, and hence the corresponding value of the nucleon energy which is to be observed. The amplitude of the emitted electronnucleon pair, defined in Eq. (2.10), is determined by the T-matrix $T_{k}$, which, according to Eq. (2.7) and Eq. (A.9), is given by 


$$
T_{k_{0}}^{(e)} \sim \int_{0}^{\infty}\left(f_{\kappa_{0}}^{(-)}\left(r_{e}\right)\right)^{\dagger}\left(\phi_{Q_{0}}^{(-)}\left(r_{n}\right)\right)^{*} v_{e n} \psi\left(r_{e} r_{n)} d r_{e} d r_{n}\right.
$$

Here $f_{\kappa_{0}}$ is a Coulomb distorted electron spinor incident on the daughter nucleus with energy corresponding to the final state, and $\psi\left(r_{e} r_{n}\right)$ is given by Eq. (2.8). In evaluating the integrals in Eq. (2.16) the two variables $r_{e}$ and $r_{n}$ are independent of each other. This expression is of the same form as given by the use of Feynman diagrams. Once the asymptotic value of the wave function is known, the corresponding differential cross section can be evaluated as is explained in the literature [18], [19].

As long as $\psi\left(r_{e} r_{n}\right)$ contains only bound state components for the nuclear wave function, such as in Eq. (2.8), the integral converges. If, however, also unbound components are included, then the integral diverges, a difficulty which has already been pointed out by Mahaux and Weidenmüller [20]. This is because now there are two particles in the continuum, whose interaction (through $v_{e n}$ in this case), extends over all regions of space. This difficulty can be seen in a three-dimensional example in which all continuum wave functions are replaced by plane waves. For the case in which $\psi$ is given by Eq. (2.8), the three dimensional integral can be done without difficulty in momentum space and yields $T_{k} \propto q^{-2} \bar{\phi}_{g}\left(\mathbf{q}+\mathbf{k}_{f}^{n}\right)$, where $\mathbf{q}=\mathbf{k}_{i}^{e}-\mathbf{k}_{f}^{e}$ is the difference between the initial and final electron momenta, $\mathbf{k}_{f}^{n}$ is the momentum of the nucleon in the final state, and $\bar{\phi}_{g}$ is the Fourier transform of the bound state wave function $\phi_{g} / r_{n}$ of the nucleon. However if $\phi_{g} / r_{n}$ is replaced by $\exp \left(i \mathbf{k}_{n}^{i} \cdot \mathbf{r}_{n}\right)$, then the corresponding result is $T_{k} \propto q^{-2} \delta\left(\mathbf{q}+\mathbf{k}_{i}^{n}-\mathbf{k}_{f}^{n}\right)$. This shows that the integrals in each partial wave component are ill behaved. Thus, if components of the wave function with two or more particles in the continuum is to be included in the overall wave function $\psi$, (such as one electron and one or more nucleons) and if the interaction between these particles is to be included, then a different formulation is required. This is commonly done in three-body calculations by going to the coordinates of relative motion and center of mass motion of the two interacting particles, and will be the object of a future paper on the (e,e' $2 \mathrm{~N})$ reaction.

The processes in which the incident electron first excites the nucleus by promoting the valence nucleon into an excited state and then knocks it out from there, can be included by 
replacing $\psi\left(r_{e} r_{n)}\right.$ in Eq. (2.16) by the sum of terms on the right hand side of Eq. (2.1). The

functions $\chi_{i}\left(r_{e}\right)$ with $i \neq g$ would be obtained to first order in $v_{e n}^{(E)}$ by the Green's function solution of Eq. (2.6a). These terms would include the effect of dispersion corrections on the $\left(\mathrm{e}, \mathrm{e}^{\prime} \mathrm{N}\right)$ reaction, but only for the bound state excitations.

In summary, this section exhibits the main ideas of how a continuum basis set for the emitted nucleon can be introduced, and the asymptotic electron wave function can be obtained by a saddle point method The expression for the (e,e'N) matrix element is identical to the usual Feynman diagram formulation, but transcends it if virtual nuclear excitation corrections are included

\section{GENERALIZATION TO THE MANY NUCLEON CASE, AND DISPERSION CORRECTIONS.}

The nuclear continuum wave function basis introduced in the previous section is unrealistic, in that the distorting potential due to the nuclear core, $V_{N}$, is real rather than complex, as would be required for an optical potential. This difficulty will now be removed by introducing internal degrees of freedom of the core nucleus, with the consequence that the inert potentials $V_{N}$ and $V_{E}$ are replaced by the microscopic interactions between the nucleons, which in turn calls for the coupled channel formalism. The orthogonality between the positive and negative energy nuclear states will still hold because these functions are eigenstates of the same Hamiltonian. The subsequent introduction of effective complex interactions, such as the optical model or effective coupling potentials, does not invalidate the orthogonality, however, inevitable errors are introduced when the non local effective interactions are replaced by approximate local ones.

The target nucleus has $A+1$ nucleons with coordinates $\mathbf{r}_{n}, \mathbf{r}_{1}, \mathbf{r}_{2}, \ldots \mathbf{r}_{A}$, and the coordinates of the bound nucleons, $\mathbf{r}_{1}, \mathbf{r}_{2}, \ldots \mathbf{r}_{A}$, are collectively denoted by $\xi$. Antisymmetrization will be ignored, the nucleon to be ejected by the incident electron will have coordinate $\mathbf{r}_{n}$, the electron coordinate is $\mathbf{r}_{e}$, and, as before, spin degrees of freedom, as well as angular 
momentum variables are ignored.

The Hamiltonian describing the nucleons is $\mathcal{H}_{A+1}\left(\mathbf{r}_{n}, \xi\right)$, the kinetic part for the relativistic Hamiltonian of the electron is again denoted by $H_{e}\left(\mathbf{r}_{e}\right)$ and hence the full Hamiltonian is

$$
\mathcal{H}=H_{e}+v_{e, A+1}+\mathcal{H}_{A+1},
$$

where

$$
v_{e, A+1}\left(\mathbf{r}_{e}, \mathbf{r}_{n}, \xi\right)=v_{e, A}\left(\mathbf{r}_{e}, \xi\right)+v^{(E)}\left(\mathbf{r}_{e}, \mathbf{r}_{n}\right)
$$

and

$$
v_{e, A}\left(\mathbf{r}_{e}, \xi\right)=\sum_{i=1}^{A} v^{(E)}\left(\mathbf{r}_{e}, \mathbf{r}_{i}\right)
$$

is the electromagnetic interaction of the electron with the nucleons in the core. In the above, $v^{(E)}(e, i)$ is the microscopic electromagnetic interaction between the electron and a nucleon which, as described in Section 2,. contains the Coulomb as well as current terms. At this stage no restrictive assumptions about the nature of the electromagnetic interaction are made. If, for example the interaction with the pion cloud (the so-called two-body currents) are included, then $v^{(E)}\left(\mathbf{r}_{e}, \mathbf{r}_{i}\right)$ does depend not only on the position and velocity of nucleon $i$ but also on the coordinates and velocities of the other nucleons. Although this possibility is implicit in the above expressions, it will not be written explicitly. Likewise, the interaction of the nucleon with the core nucleons is given by

$$
v_{n, A}\left(\mathbf{r}_{n}, \xi\right)=\sum_{i=1}^{A} v^{(N)}\left(\mathbf{r}_{n}, \mathbf{r}_{i}\right)
$$

where $v^{(N)}(n, i)$ contains both nuclear and electromagnetic interactions of nucleon $n$ with nucleon $i$.

A set of eigenstates $\Phi^{(A+1)}$ of the $A+1$ nucleon Hamiltonian is now introduced. They are of two types. The first type corresponds to the bound states of the $(A+1)$ system

$$
\mathcal{H}_{A+1} \Phi_{I}^{(A+1)}\left(\mathbf{r}_{n}, \xi\right)=\epsilon_{I}^{(A+1)} \Phi_{I}^{(A+1)}\left(\mathbf{r}_{n}, \xi\right)
$$


while the second set represents continuum states, which obey

$$
\mathcal{H}_{A+1} \Phi_{Q, i_{0}}^{(A+1,-)}\left(\mathbf{r}_{n}, \xi\right)=\epsilon_{Q, i_{0}}^{(A+1)} \Phi_{Q, i_{0}}^{(A+1,-)}\left(\mathbf{r}_{n}, \xi\right)
$$

The continuum states $\Phi_{Q, i_{0}}^{(A+1,-)}$ are the complex conjugate of the states $\Phi_{Q, i_{0}}^{(A+1,+)}\left(\mathbf{r}_{n}, \xi\right)$. For the latter one nucleon is incident with momentum $Q$ on the daughter nucleus of $A$ nucleons in a state of excitation $i_{0}$. The outgoing channels of these states contain one, two or more nucleons or clusters of nucleons in the continuum. The nuclear energy $\epsilon_{Q, i_{0}}^{(A+1)}$

$$
\epsilon_{Q, i_{0}}^{(A+1)}=\frac{\hbar^{2} Q^{2}}{2 m}+\epsilon_{i_{0}}^{(A)}+\epsilon_{s}
$$

is composed of the kinetic energy of the free incident nucleon, the excitation energy $\epsilon_{i_{0}}^{(A)}$ of the daughter nucleus in the state $i_{0}$, and the separation energy $\epsilon_{s}$ of the nucleon from the ground states of the target to that of the daughter nucleus. The recoil energy of the daughter nucleus is not written explicitly The bound states $\Phi_{I}^{(A+1)}$ and the continuum states $\Phi_{Q, i_{0}}^{(A+1,-)}$ are eigenstates of the same Hamiltonian with different energy eigenvalues, hence they are mutually orthogonal:

$$
\begin{gathered}
<\Phi_{I}^{(A+1)} \mid \Phi_{I^{\prime}}^{(A+1)}>=\delta_{I, I^{\prime}} \\
<\Phi_{I}^{(A+1)} \mid \Phi_{Q, i_{0}}^{(A+1,-)}>=0 \\
<\Phi_{Q, i_{0}}^{(A+1,-)} \mid \Phi_{Q^{\prime}, i_{0}}^{(A+1,-)}>=\frac{\pi}{2} \delta\left(Q-Q^{\prime}\right) .
\end{gathered}
$$

In the above, the ket $<\Phi_{Q, i_{0}}^{(A+1,-)} \mid$ implies complex conjugation.

The states $\Phi^{(A+1)}$ are now used as a basis set into which the complete wave function is expanded. Indicating the angular momentum algebra only very schematically one obtains for the expansion

$$
\begin{aligned}
\Psi\left(\mathbf{r}_{e}, \mathbf{r}_{n}, \xi\right)= & \frac{1}{r_{e}} \sum_{J . M_{J}}\left[\sum_{I} \chi_{I}\left(r_{e}\right) \Phi_{I}^{(A+1)}\left(\mathbf{r}_{n}, \xi\right)+\int_{0}^{\infty} \chi_{k_{Q}, i_{0}}\left(r_{e}\right) \Phi_{Q, i_{0}}^{(A+1,-)}\left(\mathbf{r}_{n}, \xi\right) d Q\right]_{J, M_{J}} \\
& \times \mathcal{Y}_{J, M_{J}}\left(\hat{\mathbf{r}}_{e}, \hat{\mathbf{r}}_{n}, \hat{\xi}\right),
\end{aligned}
$$


where $J$ and $M_{J}$ are angular momentum quantum numbers and $\mathcal{Y}_{J, M_{J}}\left(\hat{\mathbf{r}}_{e}, \hat{\mathbf{r}}_{n}, \hat{\xi}\right)$ are angular and spin functions. This expression is the many-body generalization of Eq. (2.1).

The coupled equations for the electron wave functions will now be derived, while the coupled equations for the hadron functions $\Phi_{I}^{(A+1)}$ will be discussed in section 4 . For this purpose the notation will be simplified in what follows by replacing the subscripts $I$ by $\alpha$, the subscripts $\left(Q, i_{0}\right)$ by $\beta$, i.e.,.

$$
\sum_{I} \rightarrow \sum_{\alpha} ; \int d Q \rightarrow \sum_{\beta}
$$

the combined subscript $\alpha$ and $\beta$ by $\gamma$, i.e.,

$$
\sum_{I}+\int d Q \rightarrow \sum_{\gamma}
$$

and the $(-)$ superscript on the continuum states $\Phi_{\beta}^{(A+1,-)}\left(\mathbf{r}_{n}, \xi\right)$ is suppressed. The replacement of a continuous variable by a discrete one is symbolic at this point, but it occurs naturally in numerical applications when an integral over momentum is discretized into a sum over momentum bins, as is done in the case of break-up in deuteron-nucleus scattering [14] [15].

Inserting the expansion (3.6) into the basic equation $(\mathcal{H}-E) \Psi=0$, dropping the angular momentum subscripts, multiplying on the left with $<\Phi_{\gamma}^{(A+1)} \mid$, integrating over all nuclear coordinates, and making use of the orthogonality relations (3.5), one obtains the coupled equations for the functions $\chi_{\gamma}$. For the discrete electron wave functions $\chi_{\alpha}$ the result is

$$
\left(H_{e}-E_{\alpha}^{(e)}-U_{\alpha, \alpha}^{(e)}\right) \chi_{\alpha}=-\sum_{\gamma \neq \alpha} U_{\alpha, \gamma}^{(e)} \chi_{\gamma}
$$

where

$$
U_{\alpha, \gamma}^{(e)}\left(r_{e}\right)=<\Phi_{\alpha}^{(A+1)} \mid v_{e, A+1} \Phi_{\gamma}^{(A+1)}>
$$

The equations for the continuum wave functions $\chi_{\beta}$ are

$$
\begin{aligned}
& \left(H_{e}-E_{\beta}^{(e)}+U_{\beta, \beta}^{(e)}\right) \chi_{\beta}+\sum_{\alpha} U_{\beta, \alpha}^{(e)} \chi_{\alpha}+\sum_{\beta^{\prime} \neq \beta} U_{\beta, \beta^{\prime}}^{(e)} \chi_{\beta^{\prime}} \\
= & -\sum_{\beta^{\prime}}<\Phi_{\beta}^{(A+1)} \mid v_{e, n}^{(E)} \Phi_{\beta^{\prime}}^{(A+1)}>\chi_{\beta^{\prime}},
\end{aligned}
$$


where,

$$
\begin{gathered}
U_{\beta, \beta^{\prime}}^{(e)}=<\Phi_{\beta}^{(A+1)} \mid v_{e, A} \Phi_{\beta^{\prime}}^{(A+1}> \\
U_{\beta, \alpha}^{(e)}=<\Phi_{\beta}^{(A+1)} \mid v_{e, A+1} \Phi_{\alpha}^{(A+1)}>
\end{gathered}
$$

and the electron energies appropriate to each channel are given by

$$
E_{\gamma}^{(e)}=E-\epsilon_{\gamma}^{(A+1)}
$$

Contrary to what was done in Eq. (3.7a), in Eq. (3.7b) the interaction $v_{e, n}^{(E)}$ between the two particles in the continuum was separated out and placed on the right hand side of Eq. (3.7b). The reason is that the matrix element of interaction between the two particles in the continuum will diverge, unless the wave functions in the matrix element are also distorted by that interaction. As noted before, the electron-nucleon interactions which occur in the matrix elements (3.8) are completely general, and include many-body currents.

Equations (3.7) are the multi-nucleon generalizations of Eqs. (2.6). The sum over $\gamma$ in Eq. (3.7a), contains the electromagnetic coupling to the excited bound and continuum states of the target nucleus. With exception of the term which refers to the ground state of the target nucleus, $(\alpha=g)$ these terms represent dispersion corrections to elastic and inelastic electron scattering. Some of the couplings to the bound-state excitations have been included in the literature in the calculation of dispersion corrections to elastic electron scattering by means of explicit coupled channel equations [21], [22], but explicit coupling to the continuum states, which contain the effect of the $\left(e, e^{\prime} N\right)$ reactions on the elastic or inelastic electron scattering, and which arise from the terms $\gamma=\beta$ in the sum in Eq. (3.7a), have up to now been ignored. Similarly, the second and third terms in the first line of Eq. (3.7b), with the exception of the term $\alpha=g$, also represent dispersion corrections.

The following approximations will now be made:

a) The electromagnetic coupling between the electron and the nucleon in the continuum will be ignored. This means that the terms in the second line of Eq. (3.7b) will be set to 
zero. Should it be desired to include this coupling, then the structure of the wave functions has to be modified, by making a transformation to coordinates of relative motion between the two particles in the continuum, and the coordinate of the center of mass of the two nucleons relative to the daughter nucleus. Such transformations are common in three-body calculations [19], [14], [15].

b) Dispersion correction terms will be ignored. This means that the terms on the right hand side of Eq. 3.7a) are replaced by

$$
\begin{aligned}
& \sum_{\gamma \neq \alpha} U_{\alpha, \gamma}^{(e)} \chi_{\gamma} \rightarrow U_{\alpha, g}^{(e)} \chi_{g} \text { if } \alpha \neq g \\
& \sum_{\gamma \neq \alpha} U_{\alpha, \gamma}^{(e)} \chi_{\gamma} \rightarrow 0 \text { if } \alpha=g .
\end{aligned}
$$

As a result, Eq. (3.7b) together with assumption a) reduces to

$$
\left(H_{e}-E_{\beta}^{(e)}+U_{\beta, \beta}^{(e)}\right) \chi_{\beta}=-U_{\beta, g}^{(e)} \chi_{g}
$$

Since in Eq. (3.7b) one is dealing with continuum bins, it is not clear how valid it is to ignore the bins which are the closest neighbors to bin $\beta$. This point needs further investigation. Once these terms are ignored, one obtains Eq. (3.10), which is of the form of Eqs. (2.6 b), with $B_{Q}\left(r_{e}\right)$ given by

$$
B_{Q, i_{0}}=U_{\beta, g}^{(e)} \chi_{g}=<\Phi_{Q, i_{0}}^{(A+1,-)} \mid v_{e, A+1} \Phi_{G}^{(A+1)}>f_{g}^{(+)}\left(r_{e}\right)
$$

Hence the effect of the inhomogeneous term $B_{Q, i_{0}}$ on $\chi_{\beta}$ can be carried out in the same manner as described there. In Eq. (3.11) the function $\chi_{g}$ was replaced by $f_{g}^{(+)}$, the solution of the Dirac equation for a electron elastically scattered by the target nucleus in its ground state, because, under the assumption of no dispersion corrections, $\chi_{g}$ reduces to the latter. The functions $\Phi_{Q, i_{0}}^{(A+1,-)}$ in Eq. (3.11) is quite general, but because of the choice of the (-) boundary condition, in the limit when the electron and nucleon coordinates go to infinity, only the nucleon channel associated with the daughter nucleus in state $i_{0}$ survives in the integral term in Eq. (3.6). Nevertheless, the presence of the other channels included in $\Phi_{Q, i_{0}}^{(A+1,-)}$ affects the value of $B_{Q, i_{0}}$, as will be discussed further in the next section. 
The assumption b) of ignoring dispersion corrections is not crucial to the present formulation. Their contribution to the $\left(e, e^{\prime} N\right)$ matrix element is obtained by replacing Eq. (3.11) by

$$
B_{Q, i_{0}}=\sum_{\alpha}<\Phi_{Q, i_{0}}^{(A+1,-)} \mid v_{e, A+1} \Phi_{\alpha}^{(A+1)}>\chi_{\alpha}^{(+)}\left(r_{e}\right)
$$

where to first order in the electromagnetic interaction the functions $\chi_{\alpha}$ are obtained from the solution of Eq. (3.7a), in which the sum over $\gamma$ is replaced by the single term $U_{a G}^{(e)} f_{g}^{(+)}\left(r_{e}\right)$.

In summary, the main point of the present section is to include the many-body degrees of freedom of the target nucleus by enlarging the meaning of the nuclear basis functions $\Phi_{I}^{(A+1)}$ and $\Phi_{Q, i_{0}}^{(A+1,-)}$. The rigorous calculations of these functions is impossible at this point. However, the stage is now set for making practical approximations, by writing restricted sets of coupled equations for these functions, as is explained in the next section.

\section{THE COUPLED EQUATIONS.}

The functions $\Phi_{\gamma}^{(A+1)}\left(\mathbf{r}_{n}, \xi\right)$ defined in the previous sections are general, containing zero, one, or more nucleons (or clusters) in the continuum. These functions are used in the expression (3.11) for the quantities $B_{Q . i_{0}}$, which in turn are required to obtain the asymptotic value of the electron and nucleon wave functions. In the present section an illustration is given of how to calculate the quantities $B_{Q . i_{0}}$ when the functions $\Phi_{\beta \gamma}^{(A+1,-)}$ are restricted to a subset which contains not more than one nucleon in the continuum. The coupled channel treatment is analogous to the customary case of scattering of a nucleon from a target nucleus containing $A$ nucleons. However, the present application differs from the conventional elastic scattering treatment in that the daughter nucleus can be in a highly excited state $i_{0}$, and hence the effective interactions, such as the optical model, will in principle be different from the case that $i_{0}$ represents the ground sate. There are additional reasons why the use of an optical model is not appropriate under these conditions, as will now be discussed.

The restricted set of functions $\Phi_{\gamma}^{(A+1)}$ will be denoted by 


$$
P \Phi_{\gamma}^{(A+1)}
$$

where $\mathrm{P}$ is a projection operator which eliminates all components of $\Phi_{\gamma}^{(A+1)}$ which have more than one nucleon in the continuum. The complementary projection operator is $Q$, so that $(P+Q) \Phi_{\gamma}^{(A+1)}=\Phi_{\gamma}^{(A+1)}$. The functions $P \Phi_{\gamma}^{(A+1)}$ can be expanded in terms of the bound states $\Phi_{i}^{(A)}$ of the daughter nucleus

$$
P \Phi_{\gamma}^{(A+1)}\left(\mathbf{r}_{n}, \xi\right)=\sum_{i} \frac{1}{r_{n}} \varphi_{\gamma, i}\left(\mathbf{r}_{n}\right) \Phi_{i}^{(A)}(\xi)
$$

where the expansion coefficients $\varphi_{\gamma, i}\left(\mathbf{r}_{n}\right)$ obey the usual coupled equations driven by the nucleon-nucleon interaction and where the functions $\Phi_{i}^{(A)}(\xi)$ are bound state eigenfunctions of $\mathcal{H}_{A}$

$$
\mathcal{H}_{A} \Phi_{i}^{(A)}(\xi)=\left(\epsilon_{i}^{(A)}+\epsilon_{s}\right) \Phi_{i}^{(A)}(\xi)
$$

Here $\epsilon_{i}^{A}$ is the energy of the state above the ground state, and $\epsilon_{s}$ is the separation energy of the nucleon from the ground state of the $(A+1)$ system to that of the $A$-body system. The total nuclear Hamiltonian is

$$
\mathcal{H}_{A+1}=T_{n}\left(r_{n}\right)+v_{n, A}\left(r_{n}, \xi\right)+\mathcal{H}_{A}(\xi)
$$

where $T_{n}$ is the kinetic energy operator acting on the nucleon with coordinate $r_{n}$, and $v_{n, A}$ was defined in Eq. (3.2c).

The functions $P \Phi_{\gamma}^{(A+1)}$ obey the equation

$$
\left(P \mathcal{H}_{A+1} P-E_{\gamma}^{(n)}\right) P \Phi_{\gamma}^{(A+1)}=0,
$$

where 23

$$
P \mathcal{H}_{A+1} P=T_{n}+v_{n, A}^{(e f f)}+P \mathcal{H}_{A} P
$$

and where the effective hadron interaction is given by

$$
v_{n, A}^{(e f f)}=P v_{n, A} P+P v_{n, A} Q\left(\epsilon_{\gamma}^{(A+1)}-Q \mathcal{H}_{A+1} Q+i \epsilon\right)^{-1} Q v_{n, A} P,
$$


and where

$$
E_{\gamma}^{(n)}=\epsilon_{I}^{(A+1)}-\epsilon_{i}^{(A)}-\epsilon_{s}
$$

The interaction $v_{n, A}^{(e f f)}$ differs from $v_{n, A}$ in that it includes the effect of the channels with two or more nucleons in the continuum

The coupled equations for the expansion coefficients are obtained by inserting the expansion (4.1) into Eq. (4.4), multiplying the equations on the left with $<\Phi_{i}^{(A)}(\xi) \mid$, integrating over the coordinates $\xi$, and making use of the orthogonality properties of the states $\Phi_{i}^{(A)}$. The resulting, well known, coupled equations are

$$
\left(-\frac{\hbar^{2}}{2 m} \frac{d^{2}}{d r_{n}^{2}}-E_{\gamma, i}^{(n)}\right) \varphi_{\gamma, i}\left(r_{n}\right)+\sum_{i^{\prime}} \int U_{i, i^{\prime}}^{(n)}\left(r_{n}, r_{n}^{\prime}\right) \varphi_{\gamma, i^{\prime}}\left(r_{n}^{\prime}\right) d r_{n}^{\prime}=0
$$

where

$$
U_{i, i^{\prime}}^{(n)}\left(r_{n}, r_{n}^{\prime}\right)=<\Phi_{i}^{(A)} \mid v_{n, A}^{(e f f)} \Phi_{i^{\prime}}^{(A)}>
$$

In the above the angular momentum terms such as $l(l+1) / r_{n}^{2}$ have again been left out. The potential $U_{i, i^{\prime}}^{(n)}$ is non local and complex and depends on the state $\gamma$ in view of the presence of the Green's function in Eq. (4.5b). For negative energies $\left(E_{\alpha, i}^{(n)} \leq 0\right) U_{i, i^{\prime}}^{(n)}$ should become real and the states $\varphi_{\alpha, i}$ decay exponentially at large distances. For positive energies $\left(E_{\beta, i}^{(n)}>0\right)$ the states $\varphi_{\beta, i}$ obey the (-) boundary condition, i.e., asymptotically for all states $i$ these functions are ingoing, with the exception of state $i_{0}$, for which they are both ingoing and outgoing. For this case the energy $E_{\beta, i^{\prime}}^{(n)}$ can also be expressed as

$$
E_{\beta, i^{\prime}}^{(n)}=\frac{\hbar^{2} Q_{i^{\prime}}^{2}}{2 m}=\frac{\hbar^{2} Q^{2}}{2 m}+\epsilon_{i_{0}}^{(A)}-\epsilon_{i}^{(A)}
$$

The quantities $B_{Q, i_{0}}$, defined in Eq. (3.11), will now be expressed in terms of the functions $\varphi_{\gamma, i}$. By inserting Eqs. (4.1) into Eq. (3.11), one obtains

$$
B_{Q, i_{0}}=\sum_{i}<\varphi_{\beta, i}\left(r_{n}\right) \Phi_{i}^{(A)}(\xi) \mid\left(v_{e, A+1}\right) \Phi_{G}^{(A+1)}\left(r_{n}, \xi\right)>f_{g}^{(+)}\left(r_{e}\right)
$$

where the subscript $\beta$ stands for $\left(Q, i_{0}\right)$. This is the main result of the present section. It differs from the conventional DWBA expression 


$$
B_{Q, i_{0}}=<f_{O M}^{(-)}\left(r_{n}\right) \Phi_{i_{0}}^{(A)}(\xi) \mid\left(v_{e, A+1}\right) \Phi_{G}^{(A+1)}\left(r_{n}, \xi\right)>f_{g}^{(+)}\left(r_{e}\right)
$$

in that transitions to the daughter nucleus in the final state $i_{0}$ can take place by going first to a different state $i$ through the electromagnetic coupling interaction, and from there to the final state via the strong hadron coupling effects. The probability for the latter step is given through the amplitude $\varphi_{\beta, i}$ obtained from the solution of the coupled equations (4.6a). One of these states $i$ could be an important doorway state, whose presence would spread the (e,e'p) transition strength to neighboring states, as long as the latter are coupled to the doorway state [25]. A formal justification for this point has apparently not been given previously.

Examples of the use of Eq. (4.7) are as follows. a) The effect of coupling permits the inclusion of the charge exchange process, which consists of an $\left(e, e^{\prime} n\right)$ transition followed by a $(n, p)$ process [8], [9]. b) If the state $i_{0}$ is chosen to be highly excited, which corresponds to a large missing energy, then the coupling to low-lying excitations such as hole states of valence nucleons, provides a quantitative description of how the nucleon, first emitted by knock-out from a valence orbit, excites the daughter nucleus on its way out of the nucleus. c) Coupling to excited states of the nucleon, such as a $\Delta$ excitation,.can describe the role of nucleon excitation in the (e,e'p) process. All of these effects would be missed in the DWBAoptical model formulation.

The number of states included in the sums over $i$ in Eqs. (4.6) and (4.7) can be excessively large. It is usual to replace most of the states in terms of a few "important" ones by means of expressions involving channel coupling Green's functions. As a result the states which are kept explicitly are then coupled to each other by effective interactions which are different from those of Eq. (4.5b) because they simulate the effect of a different channel space. They are non local, poorly known, and difficult to work with. In addition, the electromagnetic interaction $v_{e, A+1}$ in Eq. (4.7) will also have to be replaced by an effective interaction,

$$
v_{e f f}^{(E)}=\left[1+v_{n, A}^{(e f f)} \mathcal{G}^{\dagger}\right] v_{e, A+1}
$$

as is shown in appendix $\mathrm{C}$, and as has already been discussed [24]. The effective interaction 
$v_{\text {eff }}^{(E)}$ resembles a hybrid nucleon-nucleon $T$-matrix. It is a mixture of electromagnetic and nuclear interactions, as shown by the occurrence in Eq. $(4,8)$ of both the quantities $v_{n, A}^{(e f f)}$ and $v_{e, A+1}$. It is also non-local, in view of the occurrence of the many-body channel Green's function $\mathcal{G}^{\dagger}$.Thus, if only one state is used in Eq. (4.7), and that state is distorted by an optical potential, then the electromagnetic interaction has to be changed accordingly.

In spite of the fact that many channels other than the one being measured can contribute to $B_{k_{Q}, i_{0}}$ in Eq. (4.7), the only channel which survives asymptotically in the overall wave function is the channel $i_{0}$, since this is the only channel which has both outgoing as well as ingoing waves. This follows if one inserts the asymptotic form of the electron wave function

$$
\chi_{k_{Q}, i_{0}} \approx T_{k_{Q}}^{(e)} \exp \left(i \theta_{\kappa_{Q}}\left(r_{e}\right)\right.
$$

and the nucleon wave functions

$$
\Phi_{Q, i_{0}}^{(A+1,-)}\left(\mathbf{r}_{n}, \xi\right) \approx \sum_{j} \frac{1}{r_{n}} \frac{1}{2 i} \sum_{j}\left(H_{i_{0}}^{(+)}\left(r_{n}\right) \delta_{i_{0}, j}-H_{j}^{(-)}\left(r_{n}\right) T_{j, i_{0}}^{(N)}\right) \Phi_{j}^{(A)}(\xi)
$$

into the integral term in Eq. (3.6), and uses energy conservation to correlate the electron and nucleon wave functions. The saddle-point method of evaluating the integrals over $d Q$ then eliminates all terms but the ones which have outgoing waves in both the electron and nucleon coordinates, and only $H_{i_{0}}^{(+)}\left(r_{n}\right) \Phi_{i_{0}}^{(A)}(\xi)$ survives. One regains the asymptotic result given by Eq. (2.15), with the only difference that the inhomogeneous term $B_{Q, i_{0}}$, which

determines the asymptotic electron wave function factor $T_{k_{Q}}^{(e)}$, is now given by the more general expression (4.7).

\section{SUMMARY AND CONCLUSIONS.}

A method has been described which both justifies and criticizes the common practice of replacing the final state wave-function in the usual Feynman matrix element for the (e,e' N) reaction by a coupled channel wave function. The method points out the approximations which are implicitly made when the final state wave-function is replaced by an optical-model 
distorted wave, or when only a too restricted set of coupled equations are used. In these cases the electromagnetic electron-nucleon interaction has to be replaced by an effective interaction given by Eq. (4.8), which compensates for the approximations made in the wave function. This correction goes beyond the frequently applied Perey Factor correction, since the latter only makes up for the omitted non-localities in the optical model potential, and not, as Eq. (4.8) does, for the multi step transitions between channels, which precede or follow the electromagnetic transition. Difficulties which are due to the non-orthogonality between single particle bound states and optical model scattering states, do not occur in the coupled channel formulation, because the basic states of interest are eigenstates for positive and negative energies, respectively, of the same full hadron Hamiltonian. A further advantage of this approach is that it provides the theoretical framework for following the flow of flux from the initial channel (the ground state of the target nucleus) to the various segments of the inelastic channel space, into which the (e,e'N) strength is distributed. This information is needed to properly interpret the nuclear excitation aspect of the transparency information, obtained from (e,e' $\mathrm{N})$ or $\left(\mu, \mu^{\prime} \mathrm{N}\right)$ reactions [?].

The method developed here establishes a complete wave-function framework for describing the electron-nucleus system, so that the coupling between channels [8], [9] in the theory of the (e,e' N) reaction can be justifiably included. The basis set of hadronic wave functions has ingoing as well as outgoing nucleon waves, similar to what is the case for wave functions used in the calculation of transition matrix elements By the use of the saddle-point method in the integral over the continuum basis functions in the asymptotic limit, the ingoing part of the hadronic wave function cancels. The method is of the type commonly used in three-body calculations, but does not use the rigorous Faddeev formalism since the electromagnetic interaction between the two particles in the continuum is neglected.

The formalism is applicable, with several important modifications, to the description of the emission of two nucleons from the target nucleus. The interactions between the two nucleons in the continuum is generally ignored [27], and in order to include them a procedure can be introduced which has strong similarities to the description of the effects of deuteron 
breakup on deuteron-induced nuclear reactions [14], [15]. Experiments already point [28] to the influence of the (e,e' $2 \mathrm{~N})$ process in the emission of one nucleon, and hence a theory for this process is timely.

In summary, a derivation was given of the coupled channel wave-function description of the emission of a nucleon from a target nucleus by an incident electron. The derivation enables one to make contact with the theories of the optical model or of the coupled channel description of nucleon-nucleus reactions, and is generalizable to the case of the (e,e'2N) process.

\section{Acknowledgments.}

The author is grateful for conversations with Dr. J. J. Kelly, at the University of Mary-

land, Dr. S. Krewald at the Kernforschungs Anlage in Jülich, and several years ago, with Dr. T. W. Donnelly at MIT, concerning the feasibility of incorporating the coupled channel formalism into the description of (e,e' $\mathrm{N})$ reactions. 


\section{Appendix A:}

\section{Asymptotic solution of the inhomogeneous Dirac Eq.}

If the Dirac eq. describing the scattering on an electron from the electrostatic field of a nucleus, has also an inhomogeneous driving term, then the solution can be written in terms of a relativistic distorted Green's function acting on the inhomogeneous terms. The purpose of this Appendix is to present expressions for the asymptotic form of the result., in two different forms. 1. Involving the relativistic spinors which are the regular and irregular solutions of the distorted homogeneous Dirac equation, and 2. Involving the solutions of the Schrõdinger-like solutions of the second order equations which are equivalent to the first order Dirac equation. This second result is of value when a comparison between the relativistic and non-relativistic treatments of the distortion of the nucleons in the (e,e'N) reaction is desired.

Following the notation of Mott and Massey, [29] p. 228, a Dirac spinor of a scattering wave function, when expanded into partial waves, has two sets of radial functions for the upper and lower components. They are denoted by $G_{l}(r)$ and $F_{l}(r)$ for one set of solutions, and $G_{-l-1}(r)$ and $F_{-l-1}(r)$ for the other set. Each of these still multiplies a two-component spinor of the spherical harmonic angular functions, as indicated in Ref. [29. If one defines new radial functions $\mathcal{G}_{\lambda}(r)=G_{\lambda}(r) \times(k r)$ and $\mathcal{F}_{\lambda}(r)=F_{\lambda}(r) \times(k r)$, with $\lambda=l$ or $-l-1$, then the coupled radial Dirac equations are

$$
\begin{aligned}
& a_{-} \mathcal{G}_{\kappa}(r)-\left(\frac{d}{d r}-\frac{\kappa}{r}\right) \mathcal{F}_{\kappa}(r)=0 \\
& a_{+} \mathcal{F}_{\kappa}(r)+\left(\frac{d}{d r}+\frac{\kappa}{r}\right) \mathcal{G}_{\kappa}(r)=0
\end{aligned}
$$

where

$$
a_{ \pm}=\left[E \pm m c^{2} \pm V-e \Phi\right] / \hbar c .
$$

In the above, the quantum numbers $\kappa$ are the eigenvalues of the operator $K=-(\sigma \cdot L+\hbar)$, which assume positive or negative integer values. They are related to the angular momentum 
quantum numbers $l$ and the index $\lambda$ as follows. For $\kappa>0, \lambda=-l-1$ and $\kappa=l$. For $\kappa<0$, $\lambda=l$ and $\kappa=-l-1$. The vector spherical harmonics $\mathcal{Y}_{\kappa, \mu}$ which multiply the functions $\mathcal{G}$ and $\mathcal{F}$ are eigenfunctions of the operator $K$. The two upper and lower components of the Dirac spinors are given by $r^{-1} \mathcal{G}_{k}(r) \mathcal{Y}_{\kappa . \mu}$ and $-r^{-1} i \mathcal{F}_{-\kappa, \mu} \mathcal{Y}_{-\kappa, \mu}$, respectively [30]. In the definition of $a_{ \pm}, E$ is the total energy of the electron, $m c^{2}$ is its rest energy, $V$ is the is the scalar part of the potential, which is added to the rest mass and which is present in the case of a nucleon scattering from the field of a nucleus, and $e \Phi$ is the electrostatic Coulomb potential which is the vector part of the potential, added to the energy. The latter is negative (positive) if electrons (protons) interact with the positive charge distribution of the target nucleus. The asymptotic values of $\mathcal{G}$ are

$$
\mathcal{G}_{\kappa} \approx \sin \left(\phi_{l}+\eta_{\kappa}\right) \exp \left(i \eta_{\kappa}\right)
$$

where

$$
\phi_{l}=k r-l \pi / 2+\gamma \ln (2 k r) .
$$

The value of $l$ in Eq.(A.3b) is related to $\kappa$ as described above, $\gamma$ is the Sommerfeld parameter $Z e^{2} / \hbar v$, and $k$ is the wave number, related to the energy according to $\sqrt{(\hbar c k)^{2}+\left(m c^{2}\right)^{2}}=E$.

The inhomogeneous equations to be solved are

$$
\begin{aligned}
& a_{-} \mathcal{P}_{\kappa}(r)-\left(\frac{d}{d r}-\frac{\kappa}{r}\right) \mathcal{Q}_{\kappa}(r)=b_{\kappa}^{(U)}(r) \\
& a_{+} \mathcal{Q}_{\kappa}(r)+\left(\frac{d}{d r}+\frac{\kappa}{r}\right) \mathcal{P}_{\kappa}(r)=b_{\kappa}^{(L)}(r)
\end{aligned}
$$

Here $\mathcal{P}$ and $\mathcal{Q}$ replace the functions $\mathcal{G}$ and $\mathcal{F}$ in the homogeneous Dirac equation and $b^{(U)}$ and $b^{(L)}$ are the upper and lower components, respectively, of the inhomogeneous driving term. Asymptotically thes functions $\mathcal{P}$ and $\mathcal{Q}$ have only outgoing terms, the coefficients of which define the T-matrix elements $T_{p}$ and $T_{q}$

$$
\mathcal{P}_{\kappa} \simeq T_{\mathcal{P} \kappa} \exp \left(i \phi_{l}\right)
$$




$$
\mathcal{Q}_{\kappa} \simeq T_{\mathcal{P} \kappa} \exp \left(i \phi_{l}\right)
$$

Following the expressions given by Onley [31], for the relativistic Green's function, one obtains for the solution of Eq. (A4) the result

$$
\mathcal{P}(r)=\mathcal{G}_{R}(r) A(r)+\mathcal{G}_{I}(r) B(r)
$$

and

$$
\mathcal{Q}(r)=\mathcal{F}_{R}(r) A(r)+\mathcal{F}_{I}(r) B(r)
$$

where

$$
A(r)=\int_{r}^{\infty}\left(\mathcal{G}_{I} b^{(U)}+\mathcal{F}_{I} b^{(L)}\right) d r^{\prime}
$$

and

$$
B(r)=\int_{0}^{r}\left(\mathcal{G}_{R} b^{(U)}+\mathcal{F}_{R} b^{(L)}\right) d r^{\prime} .
$$

In the above the pair of functions $\left(\mathcal{G}_{R}, \mathcal{F}_{R}\right)$ are the regular solutions of Eqs. (A.1). with asymptotic behavior for $\mathcal{G}_{R}$ given by (A.3a). Similarly, the functions $\left(\mathcal{G}_{I}, \mathcal{F}_{I}\right)$ are the irregular solutions, with $\mathcal{G}_{I}$ normalized such that the asymptotic value is given by

$$
\mathcal{G}_{I} \approx-\frac{E+m c^{2}}{\hbar c k} \exp \left(i \phi_{l}\right) .
$$

The asymptotic behavior of the corresponding functions $\mathcal{F}$ is obtained in terms of the $\mathcal{G}$ 's from Eq. (A.1b). The relativistic equivalent of the Wronskian between the regular and irregular solutions is

$$
\mathcal{F}_{R} \mathcal{G}_{I}-\mathcal{F}_{I} \mathcal{G}_{R}=1
$$

whose value is a constant, which is equal to unity for the choice of the normalizations of $\mathcal{G}_{R}$ and $\mathcal{G}_{I}$ as described above.

As a result of the above, the asymptotic value of the spinor $(\mathcal{P}, \mathcal{Q})$, defined in Eq. (A.6), is of the form given by Eq. (A.5), with the spinor 


$$
\left(\begin{array}{c}
T_{\mathcal{P} \kappa} \\
T_{\mathcal{Q} \kappa}
\end{array}\right)=T_{\kappa}\left(\begin{array}{c}
-\left(E+m c^{2}\right) /(\hbar c k) \\
i
\end{array}\right)
$$

and

$$
T_{\kappa}=B(\infty)=\int_{0}^{\infty}\left(\mathcal{G}_{R} b^{(U)}+\mathcal{F}_{R} b^{(L)}\right) d r
$$

This is the main result of the Appendix.

The asymptotic expressions for the solution of the inhomogeneous Dirac equation will now be given in terms of the solution of the equivalent second order Schrõdinger-like equation. This procedure consists in transforming the two coupled first order differential Dirac equations for the radial partial wave functions (with or without the driving terms) into uncoupled second order differential equations, to which the usual wronskian techniques are then applied in order to extract the coefficient of the asymptotic outgoing wave. The "Darwin factors" (akin to Perey factors) which are required in this transformation (in order to eliminate the first order differential terms) in the end cancel away in the term which resembles the non-relativistic expression, but remain for the additional terms which are also present.

By differentiation and linear transformations, the first order coupled differential equations (A.1) and (A.5) are reduced to uncoupled differential equations of first and second order for $\mathcal{F}$ and $\mathcal{G}$, and similarly for $\mathcal{P}$ and $\mathcal{Q}$. The coefficients in front of the $\mathcal{G}^{\prime \prime}, \mathcal{G}^{\prime}$ and $\mathcal{G}$ are the same as the coefficients in front of $\mathcal{P}^{\prime \prime}, \mathcal{P}^{\prime}$ and $\mathcal{P}$ but in the equations for the latter the inhomogeneous terms and their derivatives also appear. Similarly for $\mathcal{F}$ and $\mathcal{Q}$. The first order terms of these equations are then eliminated by introducing the Darwin factors

$$
F_{ \pm}(r)=\sqrt{a_{ \pm}(r)}
$$

and by performing the transformation

$$
\mathcal{G}(r)=F_{+}(r) g(r) ; \mathcal{F}(r)=F_{-}(r) f(r)
$$




$$
\mathcal{P}(r)=F_{+}(r) p(r) ; \mathcal{Q}(r)=F_{-}(r) q(r)
$$

one obtains second order differential equations for $g$ and $f$, and similarly $p$ and $q$, with unit coefficients in front of the second-order derivative terms. These equations for $p$ and $q$ of course contain the inhomogeneous terms, which are denoted $b_{p}$ and $b_{q}$, respectively, but otherwise they are identical to the equations for $g$ and $f$. These transformations were already given by Darwin [29], and are routinely used in the literature [32] in order to cast the Dirac equations into Schrödinger form.

One obtains the asymptotic factor $T_{\mathcal{P}}$, defined in Eq. (A.5), by multiplying the equation for $p^{\prime \prime}$ with $g$ and the equation for $g^{\prime \prime}$ with $p$, and integrating the difference over $r$ from 0 to $\infty$. Because the coefficients in front of the $g$ and $p$ are the same, these terms cancel, and by making use of the asymptotic relations (A.3) and (A.5) one obtains

$$
\int_{0}^{\infty}\left(g p^{\prime \prime}-p g^{\prime \prime}\right) d r=-k T_{p}\left(F_{+}(\infty)\right)^{-2}
$$

The left hand side of the above equation is also equal to $\int_{0}^{\infty} g b_{p} d r$. Combining these two results, one finally obtains,

$$
\begin{aligned}
T_{p}= & \frac{E+m c^{2}}{p c} \int_{0}^{\infty} \mathcal{G} b^{(U)} d r \\
& +\frac{E+m c^{2}}{p c} \int_{0}^{\infty} \mathcal{G}\left[\left(\frac{a_{+}^{\prime}}{a_{+}}+\frac{\kappa}{r}\right) b^{(L)} / a_{+}-b^{(L) \prime} / a_{+}\right] d r
\end{aligned}
$$

By proceeding in a similar way for $\mathcal{F}$ and $\mathcal{Q}$, one obtains

$$
\int_{0}^{\infty}\left(f q^{\prime \prime}-q f^{\prime \prime}\right) d r=-i k \frac{\hbar c k}{E+m c^{2}} T_{q}\left(F_{-}(\infty)\right)^{-2}=-i T_{q}
$$

The left hand side of the above equation is also equal to $\int_{0}^{\infty} f b_{q} d r$. Combining these two results, one finally obtains,

$$
\begin{aligned}
T_{q}= & i \int_{0}^{\infty} \mathcal{F} b^{(L)} d r \\
& -i \int_{0}^{\infty} \mathcal{F}\left[\left(\frac{a_{-}^{\prime}}{a_{-}}-\frac{\kappa}{r}\right) b^{(U)} / a_{-}-b^{(U)} / a_{-}\right] d r .
\end{aligned}
$$

The first lines are the same as what one gets in the non-relativistic treatment, however, the functions which enter into these expressions are the solutions $\mathcal{G}$ or $\mathcal{F}$ of the Dirac 
equation. If one used the non-relativistic simulations $g$ or $f$ of these functions, one would first have to multiply them by the normalized Darwin factors

$$
\bar{F}_{ \pm}(r)=\sqrt{a_{ \pm}(r) / a_{ \pm}(\infty)}
$$

in order to transform them into the functions $\mathcal{G}$ or $\mathcal{F}$. These factors are many times confused with the Perey damping factors, because they have approximately the same radial dependence [33]. However these two type of factors have a different physical origin and should not be confused with each other. The terms in the second lines in Eqs. (A.13) are related to the spin-orbit potentials, since the term $\kappa / r$ is angular momentum dependent, and the factors $a_{ \pm}^{\prime} / a_{ \pm}$are the coefficients of the spin-orbit potentials in the Schrödinger form of the radial Dirac equations.

\section{Appendix B:}

\section{Asymptotic behavior via the method of steepest descent.}

In section 2 the wave function $\psi\left(r_{e}, r_{n}\right)$ was represented in terms of an integral over a continuous set of basis functions $\phi_{Q}^{(-)}\left(r_{n}\right)$, and the coefficients of the expansion, $\chi_{k_{Q}}\left(r_{e}\right)$, were obtained from the solution of an inhomogeneous Dirac equation with outgoing wave boundary conditions, $\exp \left(i k_{Q} r_{e}\right)$. Asymptotically the function $\phi_{Q}^{(-)}\left(r_{n}\right)$ has both ingoing and outgoing waves $\exp \left( \pm i Q r_{n}\right)$, and hence the asymptotic form of $\psi\left(r_{e}, r_{n}\right)$ has terms of the form $\exp \left(i k_{Q} r_{e} \pm i Q r_{n}\right)$. The two continuum variables $k_{Q}$ and $Q$ are related by energy conservation, Eq. (2.5b). As $Q$ increases, $k_{Q}$ decreases, and hence $i k_{Q} r_{e}+i Q r_{n}$ has a saddle point at $Q_{0}$ where the integral over $Q$ has a stationary phase The integral has a finite value which can be calculated by means of a steepest descent method around $Q_{0}$. On the other hand, the integral with $\exp \left(i k_{Q} r_{e}-i Q r_{n}\right)$ has no stationary phase and it vanishes. The integral involving $\exp \left(i k_{Q} r_{e}+i Q r_{n}\right)$ can be obtained from the steepest descent method which gives [Arfken and Weber] the general result

$$
\begin{aligned}
I(s) & \equiv \int_{C} g(z) \exp (s f(z) d z \\
& \approx\left(\frac{2 \pi}{\mid s f^{\prime \prime}\left(z_{0}\right)}\right)^{1 / 2} g\left(z_{0}\right) \exp \left(s f\left(z_{0}\right)+i \alpha\right) .
\end{aligned}
$$


Here $z=Q$,

$$
s f(z)=i k_{Q} r_{e}+i Q r_{n},
$$

$g(z)$ is a slowly varying function of $z, \alpha$ is a phase which depends on the direction of the path across the saddle point in the complex plane, and $s$ is the variable which becomes asymptotically large. The saddle point is determined from the requirement $d f / d z=0$, which in our case gives

$$
\frac{Q_{0}}{k_{0}}=\frac{m c^{2}}{\epsilon_{k_{0}}} x
$$

where $\epsilon_{k}$ is the energy of the electron, as defined near Eq. (2.5), $m$ is the mass of the nucleon, and $x$ is the ratio

$$
x=\frac{r_{n}}{r_{e}} .
$$

Conservation of energy, Eq. (2.5b), was used in the derivation of Eq. (B.3). From the latter one can obtain $Q_{0}$ and $k_{0}$ separately. Neglecting the rest-mass energy of the electron relative to $\epsilon_{k}$ one finds

$$
\hbar c Q_{0} \simeq m c^{2} x
$$

and

$$
\hbar c k_{0} \simeq E-m c^{2} x^{2} / 2,
$$

where $\mathrm{E}$ is the combined energy of the electron and nucleon, as given in Eq. (2.5b). This result serves to fix the ratio of distances $x$. If the electron energy $\hbar c k_{0}$ is determined from experiment, then the corresponding value of $x$ is determined from Eq. (B.5b), and the corresponding value of $Q$ from Eq. (B5.a). Inserting the value of

$$
\frac{d^{2} s f}{d Q^{2}}=-i \frac{\hbar c}{m c^{2}} r_{e}
$$

into Eq. (B.1), one obtains 


$$
I(s) \approx g\left(Q_{0}\right)\left(\frac{2 \pi i m c^{2}}{\hbar c r_{e}}\right)^{1 / 2} \exp \left[i r_{e}\left(k_{0}+\frac{m c^{2}}{\hbar c} x^{2}\right)\right]
$$

In the above, $r_{e}$ is assumed to be the independent variable, and $r_{n}$ related to it via $x$. The exponent of Eq. (B.7) can also be written as $\left.\exp \left[i r_{e} k_{0}+i r_{n} Q_{0}\right)\right]$.

\section{Appendix C. Reduction of the number of channels in terms of effective in-} teractions.

The sums over $i$ in Eqs. (4.6) and (4.7) contain a very large number of terms, which would be impossible to include in numerical calculations. Instead, it is more practical to eliminate most of the channels $i$ in terms of a few leading channels by means of channel Greens functions and effective interactions [23]. . In this Appendix the resulting modifications to the $\left(e, e^{\prime} p\right)$ formulation will be briefly indicated.

In order to simplify the notation, the potential $v_{n, A}^{(N)}$ will be replace by $v$, the matrix elements $<\Phi_{i}^{(A)} \mid v_{n, A}^{(N)} \Phi_{i^{\prime}}^{(A)}>$ by $<i|v| i^{\prime}>$, and the wave functions $\phi_{Q, i}^{\left(i_{0},-\right)}\left(r_{n}\right)$ will be replaced by $\phi_{i}\left(r_{n}\right)$. Two channels will be kept explicitly, and they will be denoted by $a$ and $b$. It is assumed that the incident wave is in channel $a$. The kinetic energy operator $-\left(\hbar^{2} / 2 m\right)\left(d^{2} / d r_{n}^{2}\right)$ is denoted by $T$, and the channel energy $E_{Q, i}$ is denoted by $E_{i}$. The coupled equations (4.4b) then take the form

$$
\begin{gathered}
\left(T-E_{i}\right) \phi_{i}+\sum_{i^{\prime}}<i|v| i^{\prime}>\phi_{i^{\prime}}=-<i|v| a>\phi_{a}-<i|v| b>\phi_{b} \\
\left(T+<a|v| a>-E_{a}\right) \phi_{a}+\sum_{i^{\prime}}<a|v| i^{\prime}>\phi_{i^{\prime}}=-<a|v| b>\phi_{b} \\
\left.T+<b|v| b>-E_{b}\right) \phi_{a}+\sum_{i^{\prime}}<b|v| i^{\prime}>\phi_{i^{\prime}}=-<b|v| a>\phi_{a}
\end{gathered}
$$

Channels $i$ can be eliminated formally [23] by using the channel-coupled Greens function $G_{i, i^{\prime}}\left(r_{n}, r_{n}^{\prime}\right)$, defined for example in Ref. [34. By using these functions in Eq. (C.1a) one obtains

$$
\phi_{i}=\sum_{i^{\prime}} G_{i, i^{\prime}}\left[<i^{\prime}|v| a>\phi_{a}+<i^{\prime}|v| b>\phi_{b}\right]
$$


where integration over the second Green's function variable is implied. Upon inserting this result into Eqs. (C.1a) and (C.1b), one obtains the "reduced" set of coupled equations

$$
\begin{aligned}
& \left(T+U_{a a}-E_{a}\right) \phi_{a}=-U_{a b} \phi_{b} \\
& \left(T+U_{b b}-E_{b}\right) \phi_{b}=-U_{b a} \phi_{a},
\end{aligned}
$$

where the effective potentials $U_{\alpha \beta}$ are given by

$$
U_{\alpha \beta}\left(r_{n}, r_{n}^{\prime}\right)=<\alpha|v| \beta>_{r_{n}} \delta\left(r_{n}-r_{n}^{\prime}\right)+\sum_{i, i^{\prime}}<\alpha|v| i>_{r_{n}} G_{i, i^{\prime}}\left(r_{n}, r_{n}^{\prime}\right)<i^{\prime}|v| \beta>_{r_{n}^{\prime}}
$$

Here $\alpha$ or $\beta$ take on the values $a$ and $b$. Because of the presence of the $G_{i, i^{i}}$ the potentials $U$ are complex and non-local, and integration over the non-localities is implied in Eqs. (C.3). One can summarize the result of Eq. (C.4) by defining an effective nucleon-nucleon potential

$$
v_{e f f}=v+v \mathcal{G}\left(r_{n}, \xi ; r_{n}^{\prime}, \xi^{\prime}\right) v
$$

where the many body channel Green's function is given by

$$
\mathcal{G}\left(r_{n}, \xi ; r_{n}^{\prime}, \xi^{\prime}\right)=\sum_{i, i^{\prime}}\left|i>_{\xi} G_{i, i^{\prime}}\left(r_{n}, r_{n}^{\prime}\right)<i^{\prime}\right|_{\xi^{\prime}}
$$

If one makes use of the result

$$
\begin{aligned}
\phi_{a}^{*}< & a\left|+\phi_{b}^{*}<b\right|+\sum_{i} \phi_{i}^{*}<i \mid= \\
& \phi_{a}^{*}\left[<a|+<a| v \mathcal{G}^{\dagger}\right]+\phi_{b}^{*}\left[<b|+<b| v \mathcal{G}^{\dagger}\right]
\end{aligned}
$$

one can rewrite the expression (4.6) for $B$ in terms of that set of channel functions $a$ and $b$ which are kept explicitly as

$$
B_{k_{Q}, i_{0}}=<\left[\phi_{Q, a}^{\left(i_{0},-\right)}\left(r_{n}\right) \Phi_{a}^{(A)}(\xi)+<\phi_{Q, b}^{\left(i_{0},-\right)}\left(r_{n}\right) \Phi_{b}^{(A)}(\xi)\right] \mid v_{e f f}^{(E)} \Phi_{G}^{(A+1)}\left(r_{n}, \xi\right)>f_{G}\left(r_{e}\right)
$$

where

$$
v_{e f f}^{(E)}=\left[1+v_{n, A}^{(N)} \mathcal{G}^{\dagger}\right] v_{e, A+1}^{(E)}
$$


This result shows that if only a few states are used in Eq. (4.6) for the calculation of the inhomogeneous term $B$, such as for example the single measured state $\mid i_{0}>$ times the corresponding optical model wave function, then, in order to make up for the contribution from the other states an effective electromagnetic interaction has to be used. This interaction is a mixture of electromagnetic and nuclear interactions, as shown by the occurrence of $v_{n, A}^{(N)} \mathcal{G}^{\dagger}$ in Eq. (C.8). 


\section{REFERENCES.}

[1] D. H. E. Gross and R. Lipperheide, Nucl. Phys. A150, 449 (1970).

[2] S. Boffi, C. Giusti, F. D. Pacati, S. Frullani, Nucl. Phys.. A319, 461 (1979).

[3] S. Boffi, C. Giusti, F. D. Pacati, Phys. Rep. 226C, 1, (1993).

[4] T. W. Donnelly and J. D. Walecka, Annu. Rev. Nucl. Sci., 25, 329 (1975); W. E. Kleppinger and J. D. Walecka, Ann. Phys. (N.Y.) A435, 679 (1985).

[5] T. W. Donnelly and A. S. Raskin, Ann. Phys. (N.Y.), 169, 247 (1986); G. Cò, A. M. Lallena and T. W. Donnelly, Nucl. Phys. A469, 684 and 716, (1987).

[6] G. Có and S. Krewald, Nucl. Phys. A433, 392 (1985); J. Ryckebusch, K. Heyde, D. Van Neck and M. Waroquier, Phys. Lett. 216B, 252 (1989); J. Ryckebusch, M. Waroquier, K. Heyde, J. Moreau and D. Ryckbosch, Nucl. Phys. A476, 237 (1988)

[7] A. Picklesimer and J. W. Van Orden, and S. J. Wallace, Phys. Rev. C32, 1312 (1985), A. Picklesimer and J. W. Van Orden, Phys. Rev. C35, 266 (1987).

[8] H. P. Blok and G. van der Steenhoven, Phys. Rev. C35, 2347 (1987).

[9] S. Jeschonnek, A. Szczurek, G. Cò, S. Krewald, Nucl. Phys. A570, 599 (1994).

[10] J. Kelly, Adv. Nucl. Phys. 23, 75 (1996)..

[11] W. Glöckle, The Quantum Mechanical Few-Body Problem (Springer Verlag, Berlin, 1983).

[12] W. Glöckle, and G. L. Payne, Phys. Rev. C45, 974 (1992).

[13] W. Glöckle, Z. Phys. 271, 31 (1974).

[14] N. Austern, Y. Iseri, M. Kamimura, M. Kawai, G. Rawitscher and M. Yahiro, Phys. Rep. 154, 
125 (1987);

[15] M. Jahiro, Y. Iseri, H. Kameyama, M. Kamimura and M. Kawai, Progr. Theo. Phys., Suppl. 89, 32, (1986); R. Singh and S.N. Mukherjee, Nuclear Reactions, (New Age International Limited Publishers, New Delhi, 1996), Ch 3.

[16] Handbook of Mathematical Functions, Ed. M. Abramowitz and I. A. Stegun, (Dover Publ. Inc., N.Y.,tenth prinitng, 1972).

[17] G. Arfken and H. J. Weber, Mathematical Methods for Physicists, (Academic Press, N.Y., 4 th. Ed., 1995), section 7.4.

[18] R. G. Newton, Ann. of Phys. (N.Y.) 74, 324 (1972).

[19] W. Glõckle, H. Witała, D. Hüber, H. Kamada and J. Golak, Adv. Nucl. Phys., in press.

[20] C. Mahaux and H. A. Weidenmüller, Shell-Model Approach to Nuclear Reactions, (American Elsevier Publ. Inc., N.Y. 1969).

[21] L. S. Cardman, D. H. Dowell, R. L. Gulbranson, D. G. Ravenhall, and R. L. Mercer, Phys. Rev. C18, 1388 (1978); G. Rawitscher, Phys. Rev. 151, 846 (1966).

[22] S. Shim, B. C. Clark, S. Hama, E. D. Cooper, R. L. Mercer, L. Ray, and G. W. Hoffmannn, Phys. Rev. C38, 1968 (1988).

[23] H. Feshbach, Theoretical Nuclear Physics: Nuclear Reactions, (John Wiley \& Sons, Inc., N.Y., 1992), Chapter IX, Section 2 J.

[24] P.M. Boucher and J.W. van Orden, Phys. Rev. C43, 582 (1991); C. R. Chinn, A. Picklesimer and J. W. van Orden, Phys. Rev. C40, 790 (1989).

[25] F. Osterfeld, Rev. Mod. Phys., 64. 491 (1992)

[26] M. R. Adams et al, Phys. Rev. Lett. 74, 1525 (1995); N. N. Nikolaev, A. Szczurek, J. Speth, J. Wambach, B.G. Zakharov, and V. R. Zoller, Phys. Rev. 50, 1296 (1994). 
[27] J. Ryckebush, M. Vanderhaegen, L. Machenil and M. Waroquier, Nucl. Phys. A568, 828, (1994); J. Ryckebush, M. Vanderhaegen, K. Heyde and M. Waroquier, Phys. Lett. B 350, 1 (1995).

[28] L. B. Weinstein et al, Phys. Rev. Lett. 64, 1646 (1990); J. B. J. M. Lanen, H. P. Blok, E. Jans, L. Lapikás, G. van der Steenhoven and P. K. A. de Witt Huberts, Phys. Rev. Lett. 64, $2250(1990)$.

[29] N. F. Mott and H. S. Massey, The Theory of Atomic Collisions, (The International Series of Monographs in Physics, Oxford University Press, London, Third Ed., 1965).

[30] The author thanks Dr. David Onley for helpful conversations about these points.

[31] D. S. Onley, Nucl. Phys. A118, 436 (1968).

[32] B. C. Clark, S. Hama and R. L. Mercer, in The Interaction Between Medium Energy Nucleons in Nuclei, - 1982, Proceedings of the Workshop on the Interaction Between Medium Energy Nucleons in Nuclei (Indiana UniversityCyclotron Facility) AIP Conf. Proc. No 97, edited by H. O. Meyer (AIP, New York, 1983), p. 260.

[33] G. H. Rawitscher, Phys. Rev. C31, 1173 (1985).

[34] G. Rawitscher, Phys. Rev. C25, 2196 (1982).

[35] Y. Jin, D.S. Onley and L.E. Wright, Phys. Rev. C45, 1311 (1992), ibid. C45, 1338 (1992). 